Conclusion Bone quality tracking should be measured relative to GA. Bone accrual seems prone to modification, especially in earlier gynaecologic ages.

\section{P2-163 MORTALITY AFTER PRENATAL EXPOSURE TO THE DUTCH FAMINE OF 1944-1945}

doi:10.1136/jech.2011.142976i.98

${ }^{1}$ L H Lumey, ${ }^{*}$ P Ekamper, ${ }^{3} \mathrm{~A} D$ Stein, ${ }^{2} \mathrm{~F}$ van Poppel. ${ }^{1}$ Columbia University, New York, New York, USA; ${ }^{2}$ NIDI, The Hague, The Netherlands; ${ }^{3}$ Emory University, Atlanta, Atlanta, USA

Introduction Some studies have examined long term health effects of famine exposure during pregnancy but little is known about possible effects on adult survival.

Methods We selected men with prenatal exposure to the Dutch famine of 1944-1945 from military examinations records $(n=408015)$ for births 1944-1946 in the Netherlands. We included men exposed in the immediate post-natal period $(n=8225)$ and in the third $(n=8197)$, the second $(n=6809)$, and the first trimester of pregnancy $(n=4666)$. We also selected men exposed around conception $(n=7727)$. Unexposed men born before or after the famine or outside the famine area were selected as controls.

Results We first linked $82 \%$ of the selected sample population $(n=45000)$ to national population records from the Netherlands Statistical Office for 2004-2009. These provide current vital status and cause of death where applicable. Successful linking was unrelated to famine exposure status or to indicators of social class. The remainder of the sample is now being traced at the Netherlands Central Bureau of Genealogy for deaths that took place prior to 2004. To date, $89 \%$ of the study population has been traced in either of these registries, covering deaths from 1967 to 2009 . Among those traced, mortality until 2009 was $9 \%$. In $24 \%$, cardiovascular disease was the primary cause of death, in $50 \%$ cancers, and in $26 \%$ other causes.

Conclusions Our findings show that long term tracing of vital status and cause of death is possible in this environment.

\section{P2-164 hUMAN PAPILLOMAVIRUS, OTHER SEXUALLY TRANSMITTED INFECTIONS AND RISK OF CERVICAL CANCER. A NORDIC JOINT STUDY}

doi:10.1136/jech.2011.142976i.99

\begin{abstract}
${ }^{1} \mathrm{~T}$ Luostarinen, ${ }^{*}{ }^{2} \mathrm{~L}$ A Dahlström, ${ }^{3} \mathrm{~K}$ Andersson, ${ }^{4} \mathrm{H}$ Ögmundsdottir, ${ }^{5} \mathrm{E}$ Jellum, ${ }^{6} \mathrm{P}$ Koskela, ${ }^{7} \mathrm{G}$ Wadell, ${ }^{8} \mathrm{M}$ Lehtinen, ${ }^{3,9} \mathrm{~J}$ Dillner. ${ }^{\prime}$ Finnish Cancer Registry, Helsinki, Finland; ${ }^{2}$ MEB, Karolinska Institutet, Stockholm, Sweden; ${ }^{3}$ Department of Laboratory Medicine, Medical Microbiology, Lund University, Malmö, Sweden; ${ }^{4}$ Cancer Research Laboratory, Faculty of Medicine, University of Iceland, Reykjavik, Iceland; ${ }^{5}$ Institute of Clinical Biochemistry, Oslo University Hospital, Rikshospitalet, Oslo, Norway; ${ }^{6}$ National Institute for Health and Welfare, Oulu, Finland; ${ }^{7}$ Department of Clinical Microbiology, Virology, Umeå University, Umeå, Sweden; ${ }^{8}$ University of Tampere, Tampere, Finland; ${ }^{9}$ Department of Laboratory Medicine, Karolinska Institutet, Stockholm, Sweden
\end{abstract}

Introduction Human papillomavirus (HPV) is considered necessary cause of invasive cervical cancer (ICC), but relations between different HPV types and other sexually transmitted infections in cervical carcinogenesis are unresolved. The CCRPB-EU Network conducted a large study, aiming to assess how major high- and lowrisk HPV types, 16, 18 and 6, and possible cofactors, Chlamydia trachomatis and herpes simplex virus type 2 (HSV-2), interact in the aetiology of cervical cancer.

Methods A case-control study was nested in four Nordic serum banks containing serum samples from approximately 1000000 women. Linkage to cancer registries resulted to 604 ICC cases diagnosed after serum sampling. Five controls were matched to bank, age at sampling and storage time. IgG antibodies specific for HPV types, $C$ trachomatis and HSV-2 were determined, and tobacco smoke exposure measured by serum cotinine, and HPV DNA in cancer tissue PCR-tested. ORs were estimated by conditional logistic regression, and adjusted for cotinine and for HPV16, HPV18 and $C$ trachomatis, when applicable.

Results Seropositivity for HPV16 did not confer any increased risk for HPV18 DNA positive cancer and HPV18 seropositivity had no association with HPV16 DNA positive cancer. HPV6 had no effect on its own but an antagonistic joint effect with HPV16. HSV-2 had little or no association. C trachomatis had a strongly increased risk for cervical cancer, which remained also among HPV18 seropositives.

Conclusions Type-specific HPV DNA persistence is important in cervical carcinogenesis. HSV-2 is possibly not a cofactor, but $C$ trachomatis is probably a strong cofactor for ICC.

\section{P2-165 UNINTENTIONAL INJURIES AT HOME AMONG PRESCHOOL CHILDREN AND EMPLOYMENT STATUS OF THEIR MOTHERS IN KERALA, INDIA}

doi:10.1136/jech.2011.142976i.100

R M Ravindran,* V Ramankutty. Achutha Menon Centre for Health Science Studies, Thiruvananthapuram, Kerala, India

Introduction Unintentional injuries are a serious public health problem worldwide as they are leading causes of death and disability in early childhood. In India, there are not many researches done in this area which hampers efforts to identify the risk factors and thus possibilities of prevention.

Objectives To find out whether there is an increased risk of sustaining unintentional injuries at home among preschool children of employed mothers.

Methods An unmatched case-control study was done in several departments of a tertiary care hospital in Trivandrum, Kerala, India. Cases were children of age $1-5$ years who attended the casualty of this hospital, with complaints of unintentional injuries where as controls included those children of the same age group who attended the same centre with complaints of illness other than injuries. Caretakers were interviewed using a pretested peer reviewed structured interview schedule. Data were entered in EpiData and analysed in SPSS version 14.

Results Mothers of 38 children (25.3\%) out of the 150 cases were employed, compared to 19 children (12.7\%) out of the 150 controls. $25 / 38$ children of working mothers who got injured were boys. Children with employed mother were found to be at a higher risk of developing unintentional injuries (OR: 2.3; 95\% CI 1.3 to 4.3 ).

Conclusion Preschool children of employed mothers are at a higher risk of sustaining injuries when compared to children of unemployed mothers. Steps should be taken to address the lack of supervision of these children during mothers' working hours. 